hen, daß die moderne Gesellschaft keineswegs gegen ihre Regression in eine Bluts-,

Abstammungs- und Schicksalsgemeinschaft gefeit ist. Nun läßt es sich nicht länger übersehen. Die Änderung der Verfassung und des Staatsangehörigkeitsgesetzes wird daran gewiß nicht viel ändern - aber getan werden muß es dennoch, und sei es auch nur, um uns selbst darüber aufzuklären, wo wir nach den Wurzeln einiger Verkehrtheiten unserer Politik zu graben haben.

Ulrich K. Preu $\beta$

\title{
Europäische und nationale Aspekte von Fremdenfeindlichkeit und Rassismus
}

Mein Diskussionsbeitrag soll auf einen europäischen und auf einen nationalen Aspekt aufmerksam machen.

I. Auch in anderen europäischen Ländern ist es in den letzten Jahren vermehrt zu fremdenfeindlichen Äußerungen und rassistischen Ausschreitungen gekommen. Vor diesem Hintergrund ist es zu erklären, daß sich im Rahmen der Europäischen Gemeinschaften das Europäische Parlament (EP), das seit seiner Gründung dem Schutz der Menschen- und Bürgerrechte eine besondere Aufmerksamkeit gewidmet hat, dieser Frage angenommen hat. Im September 1984 unterbreiteten ro9 Mitglieder des EP dem Parlamentspräsidenten den Vorschlag zur Einsetzung eines Untersuchungsausschusses zum Anwachsen von Faschismus und Rassismus in Europa, der im Oktober 1984 zusammentrat. Die Untersuchungen dieses Ausschusses, gegen dessen Tätigkeit der Abgeordnete Le Pen sogar Klage beim EuGH eingereicht hatte, sind in dem Bericht über die Ergebnisse der Arbeiten yom Dezember $19^{85}$ eindrucksvoll dokumentiert ${ }^{1}$.

Der Bericht verweist u. a. auf das Internationale Übereinkommen vom 29. 12. 1965 zur Beseitigung jeder Form von Rassendiskriminierung und die europäische Dimension der Bekämpfung von Faschismus und Rassismus durch Maßnahmen im Rahmen des Europarates. Erstmals werden die Maßnahmen der Europäischen Gemeinschaft in diesem Bereich dokumentiert. Hervorzugeben sind die »Leitlinien für eine Wanderungspolitik der Gemeinschaft « vom März 1985 , auf deren Grundlage später ein Verfahren zur vorherigen Unterrichtung und Konzertierung über die Wanderungspolitik gegenüber Drittländern eingeleitet wurde. Streitig war schon damals, in welchem Ausmaß die EG überhaupt Kompetenzen für derartige Gemeinschaftsmaßnahmen hat.

Neben zahlreichen Entschließungen des EP zum Wiederaufleben von Faschismus und Rassismus in Europa (z. B. vom 16. 1. 1986) ist auf die erstmals gemeinsam vom EP, dem Rat, den im Rat vereinigten Vertretern der Mitgliedstaaten und der Kommission verabschiedete "Erklarung gegen Rassismus und Fremdenfeindlichkeit« vom I 1.6. 1986 hinzuweisen (86/C 158/01). In der Präambel dieser Erklärung wird daran erinnert, »daß die Achtung der Menschenwürde und die Unterbindung der Rassendiskriminierung zum gemeinsamen kulturellen und rechtlichen Erbe aller Mitgliedstaaten gehören« und daß man sich des positiven Beitrags bewußt sei, »den die Arbeitnehmer aus anderen Mitgliedstaaten oder aus Drittländern zur Entwicklung des Mitgliedstaats ihres legalen Aufenthalts beigetragen haben und weiterhin beitragen können, und des Vorteils hieraus für die gesamte Gemeinschaft -«. Die Organe »verurteilen aufs schärfste alle Äußerungen von Intoleranz und Feindseligkeit sowie die Anwendung von Gewalt gegenüber einer Person oder einer Personengruppe wegen rassischer, religiöser, kultureller, sozialer oder nationaler Unterschiede«; sie »bekräftigen ihren Willen, die Persönlichkeit und

I Untersuchungsausschuß"Wiederaufleben des Faschismus und Rassismus in Europa«, Berıchterstatter: Dimitrios Evrigenis, Luxemburg 1985. 
die Würde jedes Mitglieds der Gesellschaft zu schützen und jegliche Form der Ausgrenzung von Ausiändern abzulehnen «; schließlich weisen sie »auf die Bedeutung einer angemessenen Unterrichtung und einer Sensibilisierung aller Bürger angesichts der Gefahren des Rassismus und der Fremdenfeindlichkeit« hin und heben die Notwendigkeit hervor, »dafür zu sorgen, daß jeder Akt und jede Form von Diskriminierung vermieden oder unterbunden wird «.

Das EP sah sich 1989 erneut veranlaßt, einen Untersuchungsausschuß über die Probleme des Rassismus und der Ausländerfeindlichkeit einzusetzen. Der Bericht dieses Ausschusses, der ebenso wie der vorangegangene die Situation in den einzelnen Mitgliedstaaten der EG und die Aktionen und Programme der EG ausführlich schildert, macht in erschreckender Weise deutlich, wie umfangreich und in fast allen gesellschaftlichen Bereichen verbreitet Rassismus und Ausländerfeindlichkeit wirken. ${ }^{2}$ In der jüngsten Entschließung des EP vom 30. ro. 1992 zu Rassismus, Fremdenfeindlichkeit und Antisemitismus wird erneut der Widerstand gegen jede Form von Rassismus und Ausländerfeindlichkeit bekräftigt. Die Regierungen der Mitgliedstaaten werden aufgefordert, nicht nur den Maastrichter Unionsvertrag sobald wie möglich zu ratifizieren und Vorbereitungen für eine einheitliche Einwanderungs-, Asyl- und Flüchtlingspolitik unter Achtung der Grundrechte sowie unter Einhaltung der internationalen Konventionen und Verpflichtungen zu treffen, sondern auch ein gemeinsames Aktionsprogramm gegen Rassismus und Fremdenfeindlichkeit in der Gemeinschaft aufzulegen, Initiativen von Bürgerinnen und Bürgern gegen Rassismus und Fremdenfeindlichkeit zu fördern und ihren Erfahrungsaustausch auf europäischer Ebene zu ermöglichen und ihre Vernetzung zu unterstützen (PE I61.996).

Die angesprochenen Probleme sind ein wesentlicher Grund für die Bestrebungen der EG, zu einer verbesserten Integration der Zuwanderer in den Mitgliedstaaten der EG beizutragen. Der Bericht der Kommission vom 4.9. 1990 über »Wanderungspolitiken und soziale Eingliederung der Zuwanderer in der Europäischen Gemeinschaft « (SEC(90) I 8 I 3 /endg.) verdeutlicht, wie vielfältig und umfangreich die Probleme der Integration von Zuwanderern in den Aufnahmegesellschaften sind. Dazu gehört u. a. die Ausgestaltung von bürgerlichen und politischen Rechten der Zuwanderer. Als ein wesentlicher Faktor wird die Frage des Erwerbs der Staatsangehörigkeit bzw. der Zulassung der doppelten Staatsangehörigkeit von Zuwanderern, insbesondere der Staatsangehörigen aus Staaten außerhalb der EG angesehen. Diesem Aspekt gilt meine zweite Bemerkung.

2. Unser geltendes Staatsangehörigkeitsrecht, insbesondere die Vorschriften über die Einbürgerung stehen schon seit längerem im Zentrum der ausländerrechtspolitischen Diskussion. Nicht nur Art. I I6 GG, sondern auch das Reichs- und Staatsangehörigkeitsgesetz (RuStAG) sollen nach dem Willen der Regierung geändert werden. Dies ist bislang nicht erfolgt und wird nach den jüngsten Entscheidungen der Verfassungskommission von Bundestag und Bundesrat, die zu diesen Problemen ausdrücklich keine Vorschläge machen wollte, wohl auch in absehbarer Zeit nicht erfolgen. Stein des Anstoßes ist das das deutsche Staatsangehörigkeitsrecht prägende Abstammungsprinzip (»ius sanguinis«) im Gegensatz zum Prinzip der Geburt auf dem Staatsgebiet (»ius soli«). Dementsprechend sind die Einbürgerungsvoraussetzungen eng gefaßt. Nach $\ 8$ RuStAG kann ein Ausländer auf seinen Antrag u.a. dann eingebürgert werden, wenn er einen unbescholtenen Lebenswandel geführt

2 Berıcht uber die Untersuchungsergebnisse des Untersuchungsausschusses Rassismus und Auslanderfeindlichkeit, Berıchterstatter: Glyn Ford, Luxemburg I991. Inzwischen ist die von der Kommission der EG herausgegebene vergleichende Untersuchung "Rechtsmittel zur Bekämpfung von Rassismus und Fremdenfeindlichkeit «, Luxemburg 1993, erschienen. 
hat, an dem Orte seiner Niederlassung eine eigene Wohnung oder ein Unterkommen gefunden hat und an diesem Orte sich und seine Angehörigen zu ernähren imstande ist. Die Entscheidung über die Einbürgerung steht im Ermessen der Behörde. Wie dieses Ermessen auszuüben ist, richtet sich nach den Einbürgerungsrichtlinien von $1977^{3}$. Zu den allgemeinen Grundsätzen für die Einbürgerung heißt es: "Die Bundesrepublik Deutschland ist kein Einwanderungsland; sie strebt nicht an, die Anzahl der deutschen Staatsangehörigen gezielt durch Einbürgerung zu vermehren «. In den folgenden Abschnitten dieser Einbürgerungsrichtlinien - ihre Lektüre möchte ich dringend empfehlen - werden nun die einzelnen Kriterien, anhand deren die Ermessensentscheidung zu treffen ist, näher erläutert. Ich zitiere einige markante Ausführungen, die für das vorliegende Thema von besonderem Interesse sind: Die Einbürgerung setzt eine freiwillige und dauernde Hinwendung zu Deutschland voraus; diese wird aus der nach dem bisherigen Gesamtverhalten zu beurteilenden grundsätzlichen Einstellung zum deutschen Kulturkreis zu schließen sein. Der Einbürgerungsbewerber soll insbesondere die deutsche Sprache in Wort und Schrift in dem Maße beherrschen, wie dies yon Personen seines Lebenskreises erwartet wird. Er muß nach seinem Verhalten in Vergangenheit und Gegenwart Gewähr dafür bieten, daß er sich zur freiheitlich-demokratischen Grundordnung bekennt und für ihre Einhaltung eintreten wird. Voraussetzung ist weiter die Einordnung in die deutschen Lebensverhältnisse. Dazu wird ein langjähriger, mindestens io Jahre betragender Inlandsaufenthalt für erforderlich gehalten - über die Ausnahmen von dieser Regel kann ich hier im einzelnen nicht berichten. Unter der geforderten Unbescholtenheit verstehen die Einbürgerungsrichtlinien eine einwandfreie Lebensführung; dabei genügt die Feststellung, daß über den Einbürgerungsbewerber Nachteiliges nicht bekanntgeworden ist, für sich allein nicht. Bei der Prüfung müssen vielmehr Feststellungen getroffen werden, die Aufschlüsse über den Lebensweg und das Persönlichkeitsbild des Einbürgerungsbewerbers geben. - Die hier zitierten Kriterien für die Ermessensausübung sollen verdeutlichen, inwieweit die Handhabung des Einbürgerungsermessens selbst dazu beiträgt, den Vorwurf von Rassismus und Fremdenfeindlichkeit als nicht unbegründet erscheinen zu lassen.

Abschließend möchte ich auf das für unsere Gesellschaft wohl brisanteste Problem hinweisen, nämlich die Frage nach dem Umgang mit den Angehörigen der sog. zweiten und dritten Generation der Einwanderer. Immerhin hat das neue Ausländerrecht von 1990 in $\$ \$ 85 \mathrm{ff}$. AuslG Vorschriften über die erleichterte Einbürgerung junger Ausländer vorgesehen. Während man die Einbürgerung nach $\$ 8 \mathrm{RuStAG}$ eher als Ausnahme ansehen muß, die nur bei Vorliegen eines besonderen öffentlichen Interesses angezeigt ist, weicht $\$ 8$ s AuslG für die Gruppe der im Bundesgebiet aufgewachsenen Ausländer von dieser Regel ab. Die Einbürgerung soll durch Gewährung eines Rechtsanspruchs und die Herabsetzung der Einbürgerungsvoraussetzungen gezielt gefördert werden. $\$ 85$ AuslG gibt Ausländern nach Vollendung des I6. und vor Vollendung des 23. Lebensjahres einen Rechtsanspruch auf Einbürgerung, wenn sie ihre bisherige Staatsangehörigkeit aufgeben, seit 8 Jahren rechtmäßig ihren gewöhnlichen Aufenthalt im Bundesgebiet haben, 6 Jahre im Bundesgebiet eine Schule, davon mindestens 4 Jahre eine allgemeinbildende Schule besucht haben und nicht wegen einer Straftat verurteilt worden sind. Dieser Regelanspruch ist als "Soll-Vorschrift« konzipiert, d.h. die Behörden werden im allgemeinen zum Tätigwerden unter bestimmten Voraussetzungen verpflichtet, behalten aber die Möglichkeit der Abweichung, wenn Umstände vorliegen, die den Fall als atypisch erscheinen 
lassen. Es ist bislang nicht deutlich geworden, ob die hier in sehr eingeschränkter Weise ermöglichte erleichterte Einbürgerung tatsächlich zu einer spürbaren Veränderung des Einbürgerungsverhaltens der betreffenden Angehörigen der zweiten und dritten Generation geführt hat. Eine der Hauptschwierigkeiten ist nach wie vor das Problem der doppelten Staatsangehörigkeit. Gerade männliche Jugendliche werden aus ihrer bisherigen Staatsangehörigkeit nur dann entlassen, wenn sie ihren Militärdienst absolviert oder sich mit einer entsprechend hohen Summe davon freigekauft haben (wie vor allem in den Fällen der türkischen Staatsangehörigen). Für eine erleichterte Einbürgerung sind deshalb - wie von der Bundesbeauftragten für Ausländerfragen wiederholt zur Diskussion gestellt - wirksame Maßnahmen erforderlich, die vor allem das Problem der Mehrfachstaatsangehörigkeit zum Gegenstand haben. ${ }^{4}$

Klaus Sieveking

\section{Rassismus als Problem des Völkerrechts}

Rassismus oder genauer Rassendiskriminierung stellt sich für das Völkerrecht in zwei Formen dar, die wohl zu unterscheiden sind: einmal als Diskriminierung bestimmter Bevölkerungsgruppen, zum anderen als Diskriminierung einzelner Mitglieder wegen der Zugehörigkeit zu dieser Gruppe. Auf die erste Form antwortet das Völkerrecht durch Minderheitenschutzsysteme, wie wir sie aus der Völkerbundzeit kennen. In völkerrechtlichen d.h. zwischenstaatlichen Verträgen bilateraler oder multilateraler Art verpflichten sich Staaten, Teilen ihrer Staatsanghörigen, die sich nach bestimmten Merkmalen z. B. religiöser, nationaler bzw. ethnischer Art von anderen unterscheiden, einen Sonderstatus, meist auf kulturellem Gebiet (Sprache, Schule etc.) einzuräumen. Auf dieses Problem, das seit einiger Zeit in den Vordergrund gerückt ist (die laufende Vollversammlung diskutiert eine UN-Deklaration zum Minderheitenschutz, demnächst soll ein Entwurf des Schutzes von sog. indigenous peoples behandelt werden), kann ich aus Zeitgründen nicht einghen. Ich will aber zumindest darauf hinweisen, daß die Illusion von 1945, Minderheitenprobleme seien historisch überholt, längst zerstoben ist und das Völkerrecht der gesellschaftlichen und internationalen Problementwicklung folgen muß.

Auf die zweite Form der Rassendiskriminierung antwortet das Völkerrecht durch Einräumung von Menschenrechten und deren Garantie für alle Menschen ohne Unterschiede, die ebenfalls in völkerrechtlichen Verträgen zwischen den Staaten vereinbart werden. Prominentes Beispiel ist Art. 27 des IPBürgR:

$\leadsto$ In Staaten mit ethnischen, religiösen oder sprachlichen Minderheiten darf Angehörigen solcher Minderheitn nicht das Recht vorenthalten werden, gemeinsam mit anderen Angehörigen ihrer Gruppe ihr eigenes kulturelles Leben zu pflegen, ihre eigene Religion zu bekennen um auszuüben oder sich ihrer eigenen Sprache zu bedienen."

Hier wird ein individuelles Recht eingeräumt, das allerdings nur existieren kann, wenn die Gruppe als solche in ihrer Existenz gesichert ist. Indirekt ist also ein Minderheitenschutz vorausgesetzt. Umstritten ist, ob sich diese Verpflichtung nur auf eigene Staatsangehörige des betreffenden Staates bezieht oder auch auf Ausländer auf dessen Territorium, somit auch auf Arbeitsimmigranten.

\footnotetext{
4 Im März 1993 sind von den Bundestagsfraktionen CDU/CSU, SPD und F.D.P. sowie von der Ausländerbeauftragten der Bundesregierung Gesetzesvorschläge zur Neuordnung des Staatsangehórigkeitsrechts vorgelegt worden, die auf eine vermehrte Hinnahme der Doppelstaatsangehörigkeit abziehen.
} 